\title{
ECG conduction delays: A case of viral myocarditis
}

\author{
Elizabeth Dobben, Sherene Lattimore and Julie L. Welch
}

23 yo $\mathrm{F}$ presented to the ED with 5 days of malaise, abdominal pain, vomiting, and dyspnea. Physical examination revealed tachycardia and right upper quadrant tenderness. IV fluids and symptom control were started. Her basic laboratory results were unremarkable. An Abdominal CT with IV contrast showed periportal edema and a small pericardial effusion. An ECG revealed atrial tachycardia with 2:1 conduction and a right bundle branch block. She was placed on a cardiac monitor and Cardiology was consulted. Serial ECG's revealed a widening QRS with conduction delays and eventual complete heart block on the monitor. An ECHO showed global hypokinesis with an EF of 34\%. Troponin and BNP were elevated. She underwent an emergent cardiac catheterization and pacemaker placement. In the ICU she decompensated, requiring ECMO support for 2 weeks. After a prolong hospital stay, she was discharged with and $\mathrm{EF}$ of $66 \%$ and a diagnosis of Coxsackie B viral myocarditis with cardiomyopathy.

ECG \#1 (08:02 am): Ventricular rate $64 \mathrm{bpm}$. Atrial tachycardia rate $128 \mathrm{bpm}$ with 2:1 AV conduction. Left axis deviation. Right bundle branch block with QRS $124 \mathrm{~ms}$. Left anterior fascicular block. QTc 439. ECG \#2 (09:02 am): Ventricular rate $60 \mathrm{bpm}$. Atrial tachycardia rate $133 \mathrm{bpm}$ with variable AV conduction, concerning for developing third-degree heart block. Nonspecific intraventricular block (QRS $154 \mathrm{~ms}$ ) with alternating right and left bundle branch blocks, suggesting advance conduction system disease. QTc 502. ECG \#3 (16:19 pm after cardiac catheterization and pacemaker placement): Ventricular-paced rhythm with rate 80 bpm. QRS $170 \mathrm{~ms}$. QTc $448 \mathrm{~ms}$. 


\section{References}

1. 1. Arbustini E., Agozzino M., Favalli V., Pinney S., Narula J.. MYOCARDITIS. In: Fuster V., Harrington R.A., Narula J., Eapen Z.J. eds. Hurst's The Heart, 14e New York, NY: McGraw-Hill. http://accessmedicine.mhmedical.com/content.aspx?bookid=2046\$ionid=176572086. Accessed January 16, 2019.

2. 2. Ritchie J.V., Juliano M.L., Thurman R.. ECG ABNORMALITIES. In: Knoop K.J., Stack L.B., Storrow A.B., Thurman R. eds. The Atlas of Emergency Medicine, 4e New York, NY: McGrawHill.

http://accessmedicine.mhmedical.com.proxy.medlib.uits.iu.edu/content.aspx?bookid=1763 §ionid $=125438683$. Accessed January 16, 2019. 


\section{Figures}

ECG \#1

(08:02 am): Ventricular rate $64 \mathrm{bpm}$. Atrial tachycardia rate $128 \mathrm{bpm}$ with 2:1 AV conduction. Left axis deviation. Right bundle branch block with QRS 124 ms. Left anterior fascicular block. QTc 439.

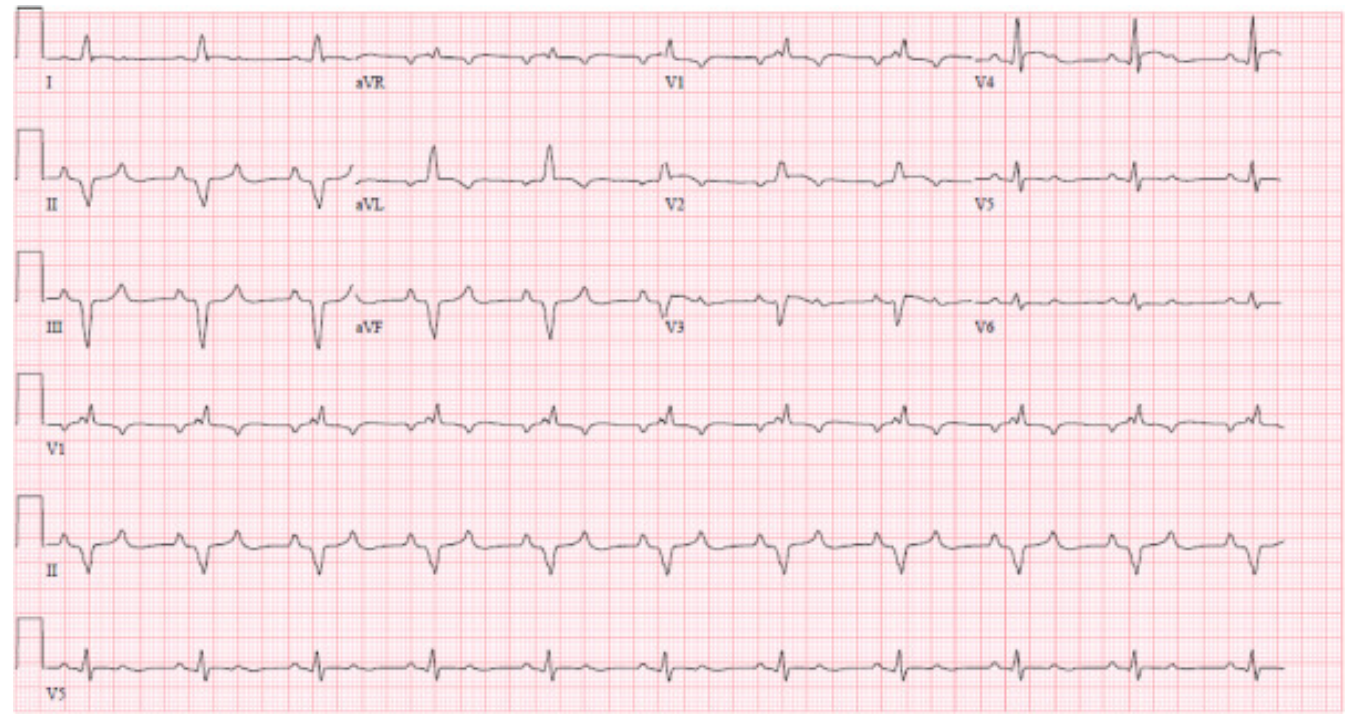




\section{ECG \#2}

(09:02 am): Ventricular rate $60 \mathrm{bpm}$. Atrial tachycardia rate $133 \mathrm{bpm}$ with variable AV conduction, concerning for developing third-degree heart block. Nonspecific intraventricular block (QRS $154 \mathrm{ms)}$ with alternating right and left bundle branch blocks, suggesting advance conduction system disease. QTc 502.

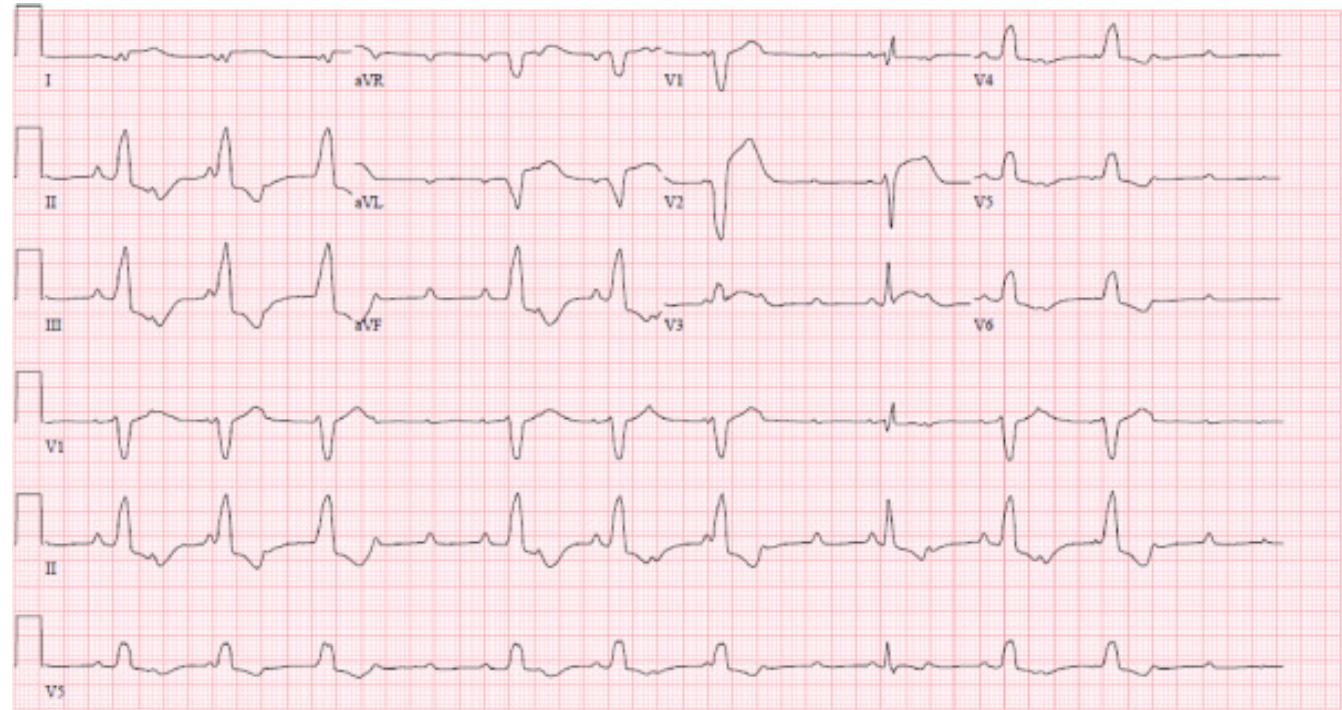


ECG \#3

(16:19 pm after cardiac catheterization and pacemaker placement): Ventricular-paced rhythm with rate $80 \mathrm{bpm}$. QRS $170 \mathrm{~ms}$. QTe $448 \mathrm{~ms}$.

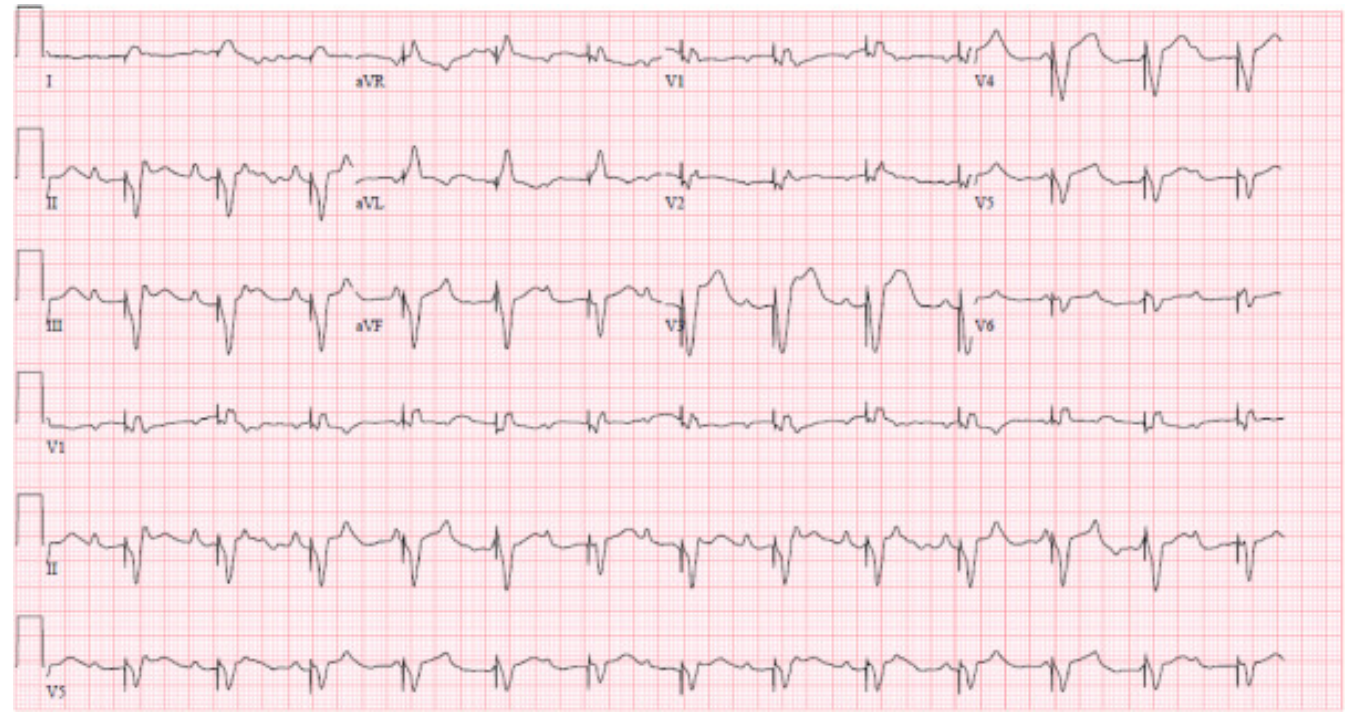

